\title{
Nurse home visits did not differ from standard care for prevention
} of recurrent child abuse

MacMillan HL, Thomas BH, Jamieson E, et al. Effectiveness of home visitation by public-health nurses in prevention of the recurrence of child physical abuse and neglect: a randomised controlled trial. Lancet 2005;365:1786-93.

In families referred to child protection agencies (CPAs), does a programme of home visiting by public health nurses (PHNs) plus standard care prevent recurrence of child physical abuse or neglect more than standard care alone?

\section{METHODS}

$\square$

Design: randomised controlled trial

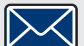

Allocation: concealed.

Blinding: blinded (data collectors and outcome assessors).

Follow up period: 2 years of intervention followed up by 1 year of observation.

Setting: 2 CPAs in Hamilton, Ontario, Canada

으 Participants: 163 English speaking families (mean age of respondent 29 y, $95 \%$ women; mean age of index child 5 y) who had been referred to the CPA for an episode of child physical abuse or neglect that occurred in the past 3 months, and the index child was $<13$ years of age and still living with the family. Cases of abuse committed by a foster parent or involving sexual abuse were excluded.

$\mathbf{R}_{\mathbf{X}}$

Intervention: 89 families were allocated to standard service arranged by the CPA plus weekly home visits of 1.5 hours by a trained PHN for 6 months; biweekly visits for the next 6 months; and monthly visits for the next year. The home visiting programme was tailored to families' individual needs and focused on intensive family support, parent education about child development, and linkage with other health and social services. 74 families were allocated to standard services alone (routine follow up by CPA caseworkers focusing on assessment of risk of recidivism, parenting education, and referral to other services).

Outcomes: subsequent episodes of physical abuse or neglect of any child in the family based on CPA reports, hospital visits because of physical abuse or neglect, and proxy (interview) measures of child maltreatment (eg, Child Abuse Potential Inventory).

Patient follow up: CPA records were available for $98 \%$ of families at 3 years (intention to treat analysis).

For correspondence: Dr $\mathrm{H} \ddot{\mathrm{L}}$ MacMillan, Departments of Psychiatry and Behavioural Neurosciences, and Pediatrics, McMaster University, Hamilton Ontario, Canada. macmilnh@mcmaster.ca

Sources of funding: Health Canada; Imperial Oil Foundation; Dr Scholl Foundation; Hamilton Social and Public Health Services Department; University of Toronto; Canadian Centre of Excellence in Child Welfare; Canadian Institutes of Health Research.

\section{MAIN RESULTS}

The home visiting and standard care groups did not differ for recurrence of physical abuse and/or neglect within the family based on CPA records, but the home visiting group had a higher rate of recurrence based on hospital visit data (table). The groups did not differ for days to first incidence of abuse or neglect (hazard ratio $0.81,95 \%$ CI 0.55 to 1.21 ) or any of the proxy measures of child maltreatment.

\section{CONCLUSION}

Home visits by public health nurses plus standard care did not differ from standard care alone for prevention of recurrent child physical abuse or neglect in families referred to child protection agencies; families who received home visits had a higher rate of abuse or neglect based on hospital visit data.

\section{Commentary}

- vidence from 2 RCTs suggests that an intensive PHN home visiting - programme that begins prenatally and continues for 2 years prevents physical abuse and neglect of children in families at risk. ${ }^{12}$ In contrast, MacMillan et al found that a visiting programme for families already involved with the child welfare system was no more effective than usual care in reducing recurrence of physical abuse and neglect. The most likely explanation for the difference in findings is that a different or more intensive intervention is required to change existing negative parenting behaviour than to prevent it. Post-hoc analysis suggested that the intervention was effective among families with less chronic CPA involvement $(<3 \mathrm{mo})$, but this hypothesis requires further study.

Strengths of the study include random allocation of participants, a theoretical basis for the intervention, training of experienced nurses, sufficient statistical power to detect between-group differences if they existed, pilot testing, psychometrically strong measures, and blinded outcome assessments.

The findings emphasise the importance of preventing physical abuse and neglect before it occurs. In jurisdictions where PHNs do not routinely begin home visits prenatally with at-risk families, perinatal, paediatric, urgent care/emergency, and primary care nurses must have a key role in identifying these families. Managers should ensure that nurses have the time and resources to effectively assess and intervene with at-risk families, communicate with other members of the healthcare team, and make appropriate early referrals. When possible, PHNs should advocate that home visits be initiated upon the birth of a child and maintained over time, particularly for families with low income or poor social support Wendy E Peterson, RN, PhD CIHR Strategic Training Postdoctoral Fellow (TUTOR-PHC) School of Nursing, University of Ottawa Ottawa, Ontario, Canada

1 Kitzman H, Olds DL, Henderson CR Jr, et al. JAMA 1997;278:644-52. 2 Olds DL, Eckenrode J, Henderson CR Jr, et al. JAMA 1997;278:637-43.

Nurse home visits plus standard care $v$ standard care alone to prevent recurrence of child physical abuse or neglect*

\begin{tabular}{|c|c|c|c|c|}
\hline Outcomes at 3 years & Home visits & Standard care alone & $\operatorname{RRR}(95 \% \mathrm{Cl})$ & NNT (Cl) \\
\hline Physical abuse (CPA records) & $33 \%$ & $43 \%$ & $23 \%(-14$ to 49$)$ & Not significant \\
\hline Neglect (CPA records) & $47 \%$ & $51 \%$ & $9 \%(-25$ to 34$)$ & Not significant \\
\hline \multirow[t]{2}{*}{ Abuse or neglect (CPA records) } & $57 \%$ & $67 \%$ & $15 \%(-9$ to 34$)$ & Not significant \\
\hline & & & RRI (Cl) & $\mathrm{NNH}(\mathrm{Cl})$ \\
\hline Abuse or neglect (hospital records) & $24 \%$ & $11 \%$ & $118 \%(6$ to 361$)$ & $8(5$ to 99$)$ \\
\hline
\end{tabular}

